\title{
Role of specific IgE on staphylococcal enterotoxin B in chronic rhinosinusitis severity
}

\author{
Suk Won Chang ${ }^{1}$, Jeong Jin Park ${ }^{1}$, Chi Sang Hwang ${ }^{1}$, Jaesung Nam ${ }^{1}$, Jong-Gyun Ha ${ }^{1}$, \\ Wasan F. Almarzouq ${ }^{1}$, Chang-Hoon Kim ${ }^{1}$, Joo-Heon Yoon ${ }^{1}$, and Hyung-Ju Cho ${ }^{1}$ \\ ${ }^{1}$ Yonsei University College of Medicine
}

July 31, 2020

\begin{abstract}
Objective: To investigate the clinical significance of specific IgE-staphylococcal enterotoxin in CRS. Design: Retrospective analysis of patients who were positive for specific IgE-staphylococcal enterotoxin B. Setting: Tertiary rhinology clinic. Participants: A total of 965 patients who were positive for specific IgE-staphylococcal enterotoxin B from December 2016 to December 2017 Main outcome measures: We retrospectively reviewed the records of 965 patients who were positive for specific IgE-staphylococcal enterotoxin B from December 2016 to December 2017. Patient demographics, titre specific IgE to staphylococcal enterotoxin B (IgE-SEB) levels, MAST, serologic test, and medical records were reviewed. Results: IgE-SEB $(\mathrm{KU} / \mathrm{L})$ was higher in CRS patients than Non-CRS patients $(0.13 \pm 0.37$ vs $0.08 \pm 0.22$, respectively; p-value: 0.044$)$, and the $\operatorname{IgE-SEB}(+,[?] 0.35)$ rate was also higher (10.06\% vs $4.46 \%$, respectively; p-value: 0.030). IgE-SEB (KU/L) was higher in the CRS group than in the fungal sinusitis group (0.13+-0.37 vs $0.03+-0.05$, respectively; p-value: $<0.001)$, and the IgE-SEB $(+,[?] 0.35)$ rate was also higher $(10.06 \%$ vs $0 \%$, respectively; p-value: 0.015$)$. Between the CRSsNP (chronic rhinosinusitis without nasal polyps) and CRSwNP (chronic rhinosinusitis with nasal polyps) groups, there were no differences in IgE-SEB $(\mathrm{KU} / \mathrm{L})$ or IgE-SEB $(+)$ rates. As the values of $\operatorname{IgE}-\mathrm{SEB}(\mathrm{KU} / \mathrm{L})$ and the IgE-SEB $(+,>0.1)$ rate increased, the CRS severity also increased. Conclusions: IgE-SEB showed a positive correlation with CRS severity but not with postoperative recurrence or nasal polyps. Further studies are needed to obtain clear evidence that IgE-SEB can be considered as an independent CRS endotype.
\end{abstract}

\section{Hosted file}

Manuscript -clinical otolaryngology.doc available at https://authorea.com/users/347717/ articles/473299-role-of-specific-ige-on-staphylococcal-enterotoxin-b-in-chronicrhinosinusitis-severity 
figures/Figure-1/Figure-1-eps-converted-to.pdf 
figures/Figure-2/Figure-2-eps-converted-to.pdf 
figures/Figure-3/Figure-3-eps-converted-to.pdf 
figures/Figure-4/Figure-4-eps-converted-to.pdf 